\title{
Structural Injury of Adjacent Organs Due to Inferior Vena Cava Filter Penetration
}

\author{
Department of Transplantation and Vascular Surgery, Korea University Anam Hospital, Seoul, Korea \\ Jun Gyo Gwon, M.D., Ph.D.
}

\begin{abstract}
A vena cava filter is an approved device for the prevention of pulmonary embolism and is commonly used in many centers. However, despite its effectiveness, certain complications have been reported, including penetration of adjacent organs by a leg of the filter. This complication is uncommon in patients in whom a vena cava filter has been inserted; nevertheless, we have experienced 3 such cases. Here, we report these cases and present a literature review of inferior vena cava filter complications and treatment methods. (Ann Phlebology 2020;18:55-58)
\end{abstract}

Key Words: Vena cava filters, Complication, Peritonitis, Duodenum, Deep vein thrombosis

\section{INTRODUCTION}

The inferior vena cava (IVC) filter is effective in the prevention of pulmonary embolism (PE) for the management of a venous thromboembolic (VTE) event (1). However, in $4 \% \sim 11 \%$ of cases, IVC filter-related complications, such as bleeding at the insertion site, malfunction of filter deployment, malposition of the filter, filter migration with or without filter fracture, and proximal migration to the right ventricle or pulmonary artery, have been reported $(2,3)$. Rarely, a leg of the filter may perforate the IVC wall and further penetrate adjacent structures including the duodenum or pancreas.

Here, we report 3 cases of complications with IVC filters, which were required to be removed and open surgical repair

\footnotetext{
Corresponding author : Jun Gyo Gwon, 73 Goryudae-ro, Seongbuk-gu, Seoul 02841, Korea, Department of Transplantation and Vascular Surgery, Korea University College of Medicine

Tel: 02-920-5978, Fax: 02-928-1631

E-mail: doctorgjg@gmail.com
}

This study is supported by a Korea University Medical Center Grant (No. O2000671). carried out due to filter penetration into adjacent structures: the pancreas, the duodenum, and the periaortic tissue. We also provide a literature review on the incidence of IVC filter complications.

\section{CASE REPORT}

\section{1) Case 1}

A 34-year-old woman had complained of epigastric pain for 1 month. Her symptoms were exacerbated by eating. She had no fever, hematochezia, hematemesis, or melena, and no medical history except for IVC filter insertion. Three years ago, she was diagnosed with right iliac vein thrombosis with a floating IVC thrombus. A retrievable IVC filter was inserted into the infra-renal IVC, and anticoagulation drugs were administered. After a few weeks, she underwent angiography for the removal of the IVC filter; however, a thrombus was located on the filter, and thus it was not removed to prevent pulmonary thromboembolism.

Her primary care physician considered duodenal ulceration as a cause of the epigastric pain, and she underwent an esophagogastroduodenoscopy (EGD). EGD revealed a sharp wire in the third part of the duodenum (Fig. 1), but there was no bleeding or ulceration. Subsequently, she underwent open surgical removal of the IVC filter. During the operation, it was found that the legs of the IVC filter were covered by IVC endothelium. It was impossible to simply remove the filter, and the IVC wall was partially resected. Following removal of the filter, the perforated duodenum and IVC wall were repaired by primary closure. On postoperative day 5, there was no abdominal pain or leukocytosis after a soft diet. She was discharged without any complications.

2) Case 2

A 75-year-old woman visited the hospital for acute left 


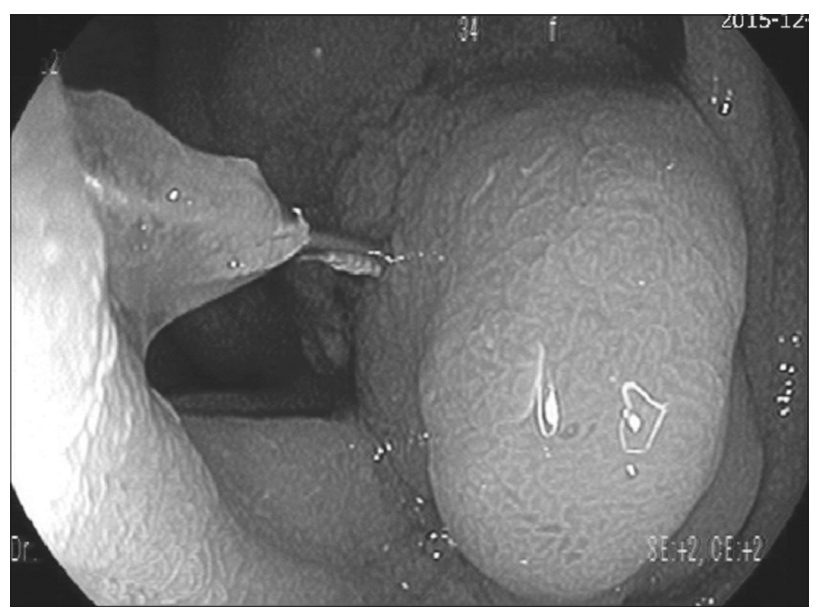

Fig. 1. Penetration of an IVC filter leg into the duodenal lumen without the presence of inflammation or ulceration.

leg edema and calf pain. She was diagnosed with a deep vein thrombosis in the left common femoral vein. As a result, an IVC filter was inserted through the right internal jugular vein. Two weeks later, she complained of sharp periumbilical pain and presented with leukocytosis (WBC 13500/ $\mu \mathrm{l}$ ). A computed tomography (CT) scan revealed penetration of a leg of the IVC filter into the head of the pancreas (Fig. 2). She was transferred to our center. Endoscopic retrograde cholangiopancreatography was conducted and pancreatic duct injury was ruled out. During the operation, severe inflammatory changes were observed near the IVC wall; however, there was no adhesion between the IVC filter and the interior of the IVC wall. The filter was removed easily. On postoperative day 3, a soft diet was tolerable, and she was discharged without any complications.

\section{3) Case 3}

A 39-year-old woman visited the hospital for a chronic cough and dyspnea. A CT scan was conducted for evaluation, revealing a pulmonary embolism in both main pulmonary arteries. She underwent a pulmonary endarterectomy using an infra-renal-type IVC filter. The IVC filter was not removed to prevent pulmonary thromboembolism. She had thrombogenic conditions because of protein $\mathrm{C}$ deficiency. She was discharged without complications; however, 3 months later, she suffered from intermittent abdominal pain. There was no evidence of fever, leukocytosis, or gastrointestinal symptoms other than abdominal pain. We performed a CT scan for evaluation, and the radiological results showed a leg of the IVC filter on the adjacent tissue. A laparotomy

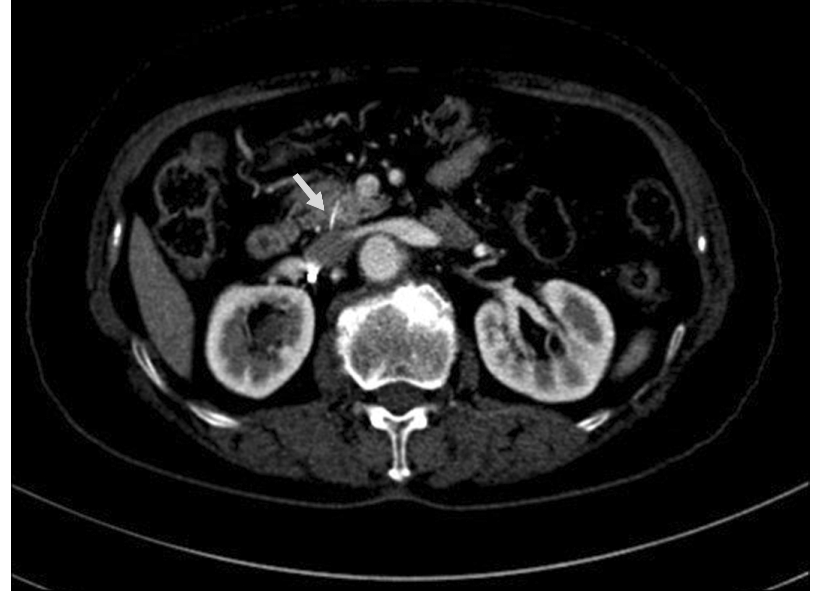

Fig. 2. CT scan revealing an IVC filter leg projecting into the head of the pancreas (yellow arrow is the head of the pancreas).

was conducted for retrieval of the IVC filter. During the operation, the leg of the filter was found to be spurred the periaortic tissue and lymph nodes; however, there was no injury to the other adjacent organs. The IVC filter was easily removed through a longitudinal incision in the IVC wall, and she was discharged without any complications or abdominal pain.

\section{DISCUSSION}

Since the 1970s, the vena cava filter has been used for the prevention of pulmonary embolism in patients with VTE, and retrievable IVC filters have been used since the 2000s.

Indications for IVC filter insertion are not rigid. One indication is repeated VTE occurrence despite adequate anticoagulation medication. Another is a patient diagnosed with VTE who is not able to take anticoagulation medication. However, lack of established indications creates a problem. For example, in case 2, the patient with an IVC filter was transferred to our clinic, but we were unable to identify the reason for the insertion. Thus, unnecessary IVC filter insertions should be avoided to reduce IVC filter complications, and strict indications should be in place.

There are several complications related to inserted IVC filters; Jehangir et al. categorized these as insertion-related, device failure, and late complications (4). During IVC filter insertion, the complication rate is believed to be less than $0.5 \%$ (5), with complications including hemorrhage, pneumothorax, filter misplacement, excessive tilt of the filter, and vessel injury. Late complications include recurrent pulmonary embolism, IVC occlusion, filter migration, and filter 
leg perforation.

Following the introduction of the retrievable IVC filter, the complication rate should have diminished. However, in reality, only $50 \%$ of retrievable IVC filters are removed from patients, with the majority remaining asymptomatic and without clinical consequence (4).

Vena cava perforation is one of the main complications of IVC filters, being reported in up to $40 \%$ of cases. Interestingly, almost all these cases are asymptomatic and found only incidentally (6).

All 3 of the cases in this study used a Celect IVC filter (Cook, Inc., Bloomington, Indiana). Ryu et al. reported a $43 \%$ penetration rate of this filter and that of the Option IVC filter (Rex Medical, Conshohocken, Pennsylvania) was $0 \%(7)$.

The mechanism of IVC perforation remains unclear; however, the shape of the legs and duration of the placement of the filter, and filter tilting during placement, are thought to be important risk factors (4). In all 3 cases reported here, the filters were tilted, and these were symptomatic cases, where the penetrated leg was piercing adjacent structures; the duodenum, the pancreas, and periaortic tissue.

We removed all the IVC filters during open surgical repair due to the confirmation of structural injury of adjacent tissue. In case 1 , the leg of the IVC filter penetrated the duodenal posterior wall at the third portion. The leg was removed from the duodenal wall and the injured duodenum was repaired. In case 2 , the leg of the IVC filter was placed in the tissue of the head of the pancreas; however, following removal of the leg and close inspection of the pancreatic tissue, there was no injury.

All the filters were removed through an inferior vena cava incision. Open surgical removal of the IVC filters showed good outcomes with a low complication rate in all cases, with either extraction of the filter or removal of the offending legs (8). In case 1 , the legs of the filter were covered by endothelium and fixed on the IVC wall, without meticulous dissection through endovascular retrieval, there was a chance of vascular injury or hemorrhage around the IVC filter (Fig. 3).

In conclusion, IVC filters decrease the mortality rate of venous thromboembolism patients; While complication is not common, it is possible and serious in particular perforation of the legs of the filter through the IVC wall, causing injury to adjacent structures. An improved device is required for the stable and safe placement of filters, and

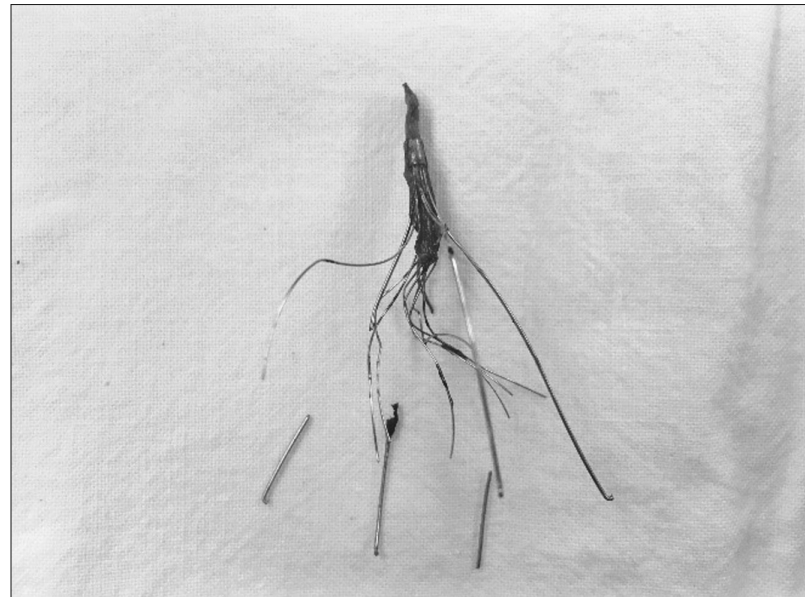

Fig. 3. Removal of the IVC filter by open surgical treatment. Filter was covered by IVC endothelium.

most of all, it is important that implanting clinicians take great care of patients with retrievable IVC filters by removing the filter as soon as it is no longer needed (9).

\section{CONFLICT OF INTEREST}

This research did not receive any specific grant from funding agencies in the public, commercial, or not-for-profit sectors.

\section{REFERENCES}

1) Decousus H, Leizorovicz A, Parent F, Page Y, Tardy B, Girard $\mathrm{P}$ et al. A clinical trial of vena caval filters in the prevention of pulmonary embolism in patients with proximal deep-vein thrombosis. Prevention du Risque d'Embolie Pulmonaire par Interruption Cave Study Group. N Engl J Med. 1998;338:409-15.

2) Vandy F, Rectenwald JE, Criado E. Late gastrointestinal complications of inferior vena cava filter placement: case report and literature review. Perspect Vasc Surg Endovasc Ther. 2011;23:261-4.

3) Shang EK, Nathan DP, Carpenter JP, Fairman RM, Jackson BM. Delayed complications of inferior vena cava filters: case report and literature review. Vasc Endovascular Surg. 2011;45:290-4.

4) Jehangir A, Rettew A, Shaikh B, Bennett K, Jehangir Q, Qureshi A et al. IVC filter perforation through the duodenum found after years of abdominal pain. Am J Case Rep. 2015;16:292-5.

5) Harvey JJ, Hopkins J, McCafferty IJ, Jones RG. Inferior vena cava filters: what radiologists need to know. Clin 
Radiol. 2013;68:721-32.

6) Miyahara T, Miyata T, Shigematsu K, Deguchi J, Kimura $\mathrm{H}$, Ishii $\mathrm{S}$ et al. Clinical outcome and complications of temporary inferior vena cava filter placement. J Vasc Surg. 2006;44:620-4.

7) Ryu RK, Desai K, Karp J, Gupta R, Evans AE, Rajeswaran $\mathrm{S}$ et al. A Comparison of Retrievability: Celect versus Option Filter. J Vasc Interv Radiol. 2015;26:865-9.
8) Malgor RD, Labropoulos N. A systematic review of symptomatic duodenal perforation by inferior vena cava filters. J Vasc Surg. 2012;55:856-61 e3.

9) Durack JC, Westphalen AC, Kekulawela S, Bhanu SB, Avrin DE, Gordon RL et al. Perforation of the IVC: rule rather than exception after longer indwelling times for the Gunther Tulip and Celect retrievable filters. Cardiovasc Intervent Radiol. 2012;35:299-308. 